\title{
Design and Implementation of a Fully Asynchronous SFQ Microprocessor: SCRAM2
}

\author{
Yusuke Nobumori, Takanobu Nishigai, Kazunori Nakamiya, Nobuyuki Yoshikawa, Akira Fujimaki,
} Hirotaka Terai, and Shinich Yorozu

\begin{abstract}
A microprocessor test vehicle was developed for the investigation of asynchronous design methodology for rapidsingle-flux-quantum (RSFQ) circuits. We have designed and implemented a fully asynchronous RSFQ microprocessor, named SCRAM2. The data-driven self-timing (DDST) architecture is used for the design of circuit blocks of the SCRAM2. In order to ensure the logical ordering between the circuit blocks, bit-serial handshaking was adopted. The performance of the handshaking system was enhanced based on the scalable-delay-insensitive (SDI) model. The SCRAM2 is an 8-bit bit-serial microprocessor with three-stage pipelining, with a basic microarchitecture similar to that of our previously designed synchronous microprocessor, CORE1 $\alpha$. The estimated average performance of the SCRAM2 is 577 MIPS using a logic simulation. We have implemented all circuit components using the SRL $2.5 \mathrm{kA} / \mathrm{cm}^{2} \mathrm{Nb}$ process and confirmed their correct operation. Several operations of the SCRAM2 have been successfully confirmed.
\end{abstract}

Index Terms-Asynchronous logic circuits, Josephson logic, SFQ circuit, superconducting integrated circuits.

\section{INTRODUCTION}

$\mathbf{R}$ APID-SINGLE-FLUX-QUANTUM (RSFQ) circuits are promising candidates for future high-performance LSI technology, because of their high-speed operation and low power consumption [1]. Several groups have recently been developing large RSFQ digital systems [2]. The successful demonstration of a RSFQ microprocessor, named CORE $1 \alpha$, has been reported [3]. At present, a synchronous approach is generally used in designing RSFQ digital systems. However, the pico-second timing design of globally synchronous RSFQ digital systems operating at clock frequencies beyond several tens of $\mathrm{GHz}$ is an awkward and time consuming task. In order to solve the timing issues in synchronous RSFQ circuits, we have been investigating several asynchronous design approaches, i.e. data-driven self-timing (DDST) [4] and binary decision

Manuscript received August 29, 2006. This work was supported by the New Energy and Industrial Technology Development Organization (NEDO) through ISTEC as Collaborative Research and Superconductors Network Device Project.

Y. Nobumori, T. Nishigai, K. Nakamiya, and N. Yoshikawa are with the Department of Electrical and Computer Engineering, Yokohama National University, Yokohama, 240-8501 Japan (e-mail: yoshi@yoshilab.dnj.ynu.ac.jp).

A. Fujimaki is with the Department of Quantum Engineering, Nagoya University, Nagoya, 464-8603 Japan (e-mail: fujimaki@ nuee.nagoya-u.ac.jp).

$\mathrm{H}$. Terai is with the National Institute of Information and Communication Technology, Kobe, 651-2492 Japan (e-mail: terai@ nict.go.jp).

S. Yorozu is with the International Superconductivity Technology Center Superconductivity Research Laboratory, Tsukuba, 305-8501 Japan (e-mail: yorozu@istec.or.jp).

Color versions of one or more of the figures in this paper are available online at http://ieeexplore.ieee.org.

Digital Object Identifier 10.1109/TASC.2007.898658



Fig. 1. Asynchronous design approaches used in the SCRAM2.

diagram (BDD) [5] at the circuit level, and vector or bit-serial handshaking at the system level [4], [6].

In this study, we have designed a fully asynchronous microprocessor, named SCRAM2, to investigate the effectiveness of our asynchronous design approaches in the large RSFQ digital system. The SCRAM2 is an 8-bit microprocessor based on bit-serial architecture with a basic microarchitecture similar to that of our previously designed synchronous microprocessor, CORE $1 \alpha$. The data path of the SCRAM2 is pipelined into three stages using a handshake protocol. In this paper, we describe the asynchronous design methodologies and microarchitecture of the SCRAM2. The SCRAM2 is also implemented and tested and its performance evaluated.

\section{Asynchronous Design Methodology IN SCRAM2}

In our asynchronous design methodology, the entire system is divided into circuit blocks of a reasonable size as illustrated in Fig. 1. Each circuit block has dual-rail inputs and outputs, and is designed based on asynchronous design approaches, such as DDST or BDD.

The DDST approach [4] uses conventional synchronous RSFQ clocking just inside the circuit block, where the local clock is generated from dual-rail signal inputs using a logical OR gate. At the output of the circuit block, dual-rail signals are reproduced using a flip-flop with complementary outputs. In the BDD approach [5], a BDD, which is a directed graph 
representing a logical function, is directly implemented using one to two RSFQ switches. The answer of the BDD is obtained by tracing a BDD tree from top to bottom, according to the state of each node. In both asynchronous approaches, the input and output of the circuit block are a dual-rail, therefore operation of the circuit is data-driven and no global clock is required outside the circuit block.

In order to prevent data collision between the circuit blocks and to ensure logical ordering we have adopted a handshake protocol. A large size overhead and delay is generally a disadvantage for a handshaking system. However, in our approach, the handshaking overhead is relatively reduced by adopting bitserial handshaking, where the data transfer is performed by a unit of bit-serial data during a single handshaking process [4], [6]. Through the use of these asynchronous approaches, global clocks have been completely eliminated in the microprocessor.

\section{Delay Model for Asynchronous Circuits}

There are several delay models in the asynchronous design, which are based on assumptions for gate and wire delays in the system. In the delay-insensitive (DI) or quasi-delay-insensitive (QDI) model, the gate and wire delays are finite but unbounded, i.e. no upper limit is assumed in the delay [7]. In order to guarantee completion of the circuit operation, completion signals have to be calculated from the output of the circuit component, resulting in delay and increased hardware cost. However, with a scalable-delay-insensitive (SDI) model, the gate and wire delay is unbounded, but the relative delay ratio between any two components is bounded [8].

The SDI model is defined as follows: The delay of any two components, $\mathrm{C}_{1}$ and $\mathrm{C}_{2}$, in the system is assumed to be $d_{1}$ and $d_{2}$, respectively. The relative delay $D$, of $\mathrm{C}_{1}$ and $\mathrm{C}_{2}$, is expressed as $D=d_{2} / d_{1}$. The relative delay, estimated by simulation, is defined as $D_{\mathrm{e}}$, whereas the experimental relative delay, i.e. the actual relative delay, is $D_{\mathrm{a}}$. Therefore, the relative variation ratio, $R=D_{\mathrm{a}} / D_{\mathrm{e}}$, of any two components is restricted by

$$
1 / K<R<K
$$

for the SDI model, where $K$ is a constant and is called the maximum variation ratio.

In the SDI model, it is guaranteed that the operation of $\mathrm{C}_{1}$ is completed earlier than that of $\mathrm{C}_{2}$ if the following relation is satisfied.

$$
d_{2}>K \bullet d_{1}
$$

Fig. 2 compares the delays in detection of completion for the QDI and SDI models. In the QDI model, it takes a 13-unit delay to produce the completion signal, QDI ACK. On the other hand, for the SDI model, because the SDI ACK is produced in parallel with the operation of the functional block, the time required for SDI ACK to be issued is an 8 unit delay. If we assume $d_{1}$ and $d_{2}$ are the delay of the functional block and the completion detector (CD), respectively, and $K=1.5$, then (2) is satisfied, which means that the completion of the functional block is guaranteed to precede the QDI ACK.

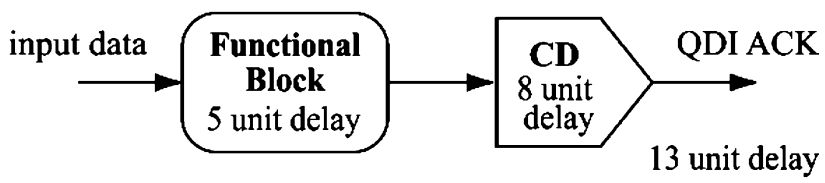

(a)

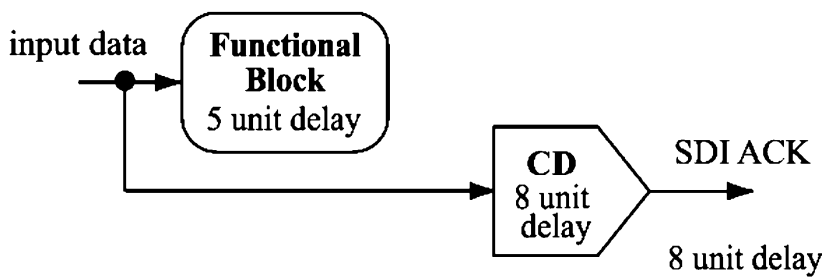

(b)

Fig. 2. Detection of the completion signals in (a) QDI and (b) SDI models. The $\mathrm{CD}$ represents the completion detector.

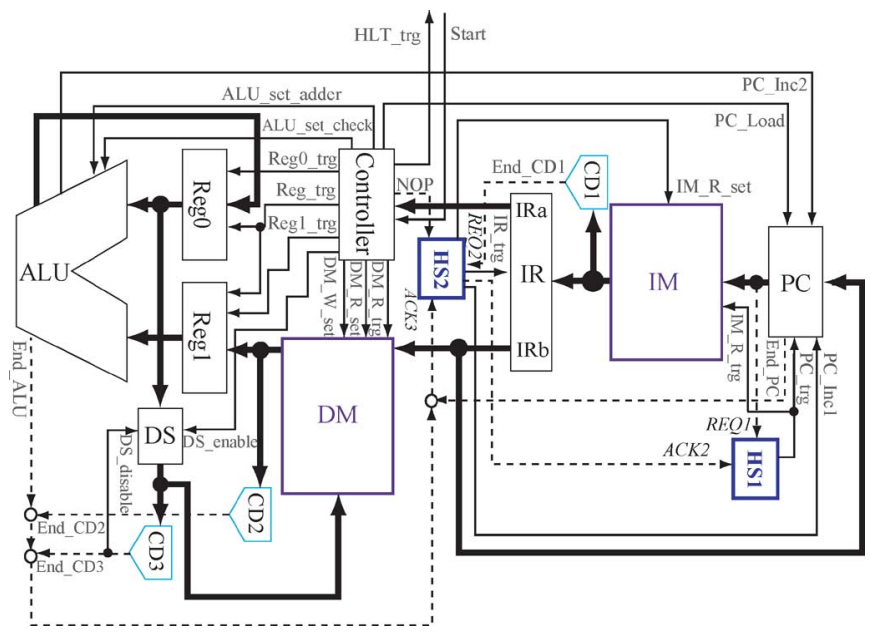

Fig. 3. Block diagram of the SCRAM2. The bold lines represent data or instruction paths. The thin solid lines represent the control signals and the dashed lines show the completion signals of each pipeline stage.

\section{MicROARCHITECTURE OF SCRAM2}

The microarchitecture of SCRAM2 is similar to that of our synchronous version of the RSFQ microprocessor CORE1 $\alpha$. The SCRAM2 is an 8-bit pipelined bit-serial microprocessor based on the complexity reduced (CORE) concept [9], which reduces the complexity of a system using high throughput of RSFQ circuits. Fig. 3 shows a block diagram of the SCRAM2. It is mainly composed of a controller, an 8-bit instruction register (IR), a 5-bit program counter (PC), a bit-serial arithmetic and logic unit (ALU), two 8-bit registers (Reg0 and Reg1), three completion detectors (CD1, CD2 and CD3), a data memory (DM), and an instruction memory (IM). Two handshaking circuits (HS1 and HS2), which provide trigger signals when an acknowledge signal (ACK) and a request signal (REQ) are received from the next and previous pipeline stages, respectively, control the three-stage asynchronous pipelining.

Fig. 4 shows a state transition diagram of the SCRAM2. Instructions are executed throughout three stages, which correspond to instruction addressing, instruction fetch, and execution. The state transition between pipeline stages is controlled by the handshake protocol. For example, the transition from stage 


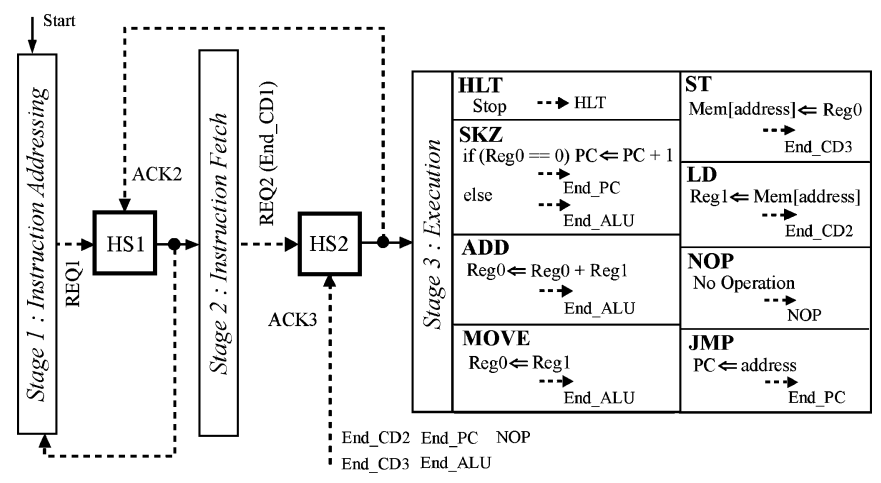

Fig. 4. State transition diagram of the SCRAM2. Solid lines represent trigger signals for the next pipeline stage, and dashed lines are completion signals from each pipeline stage.

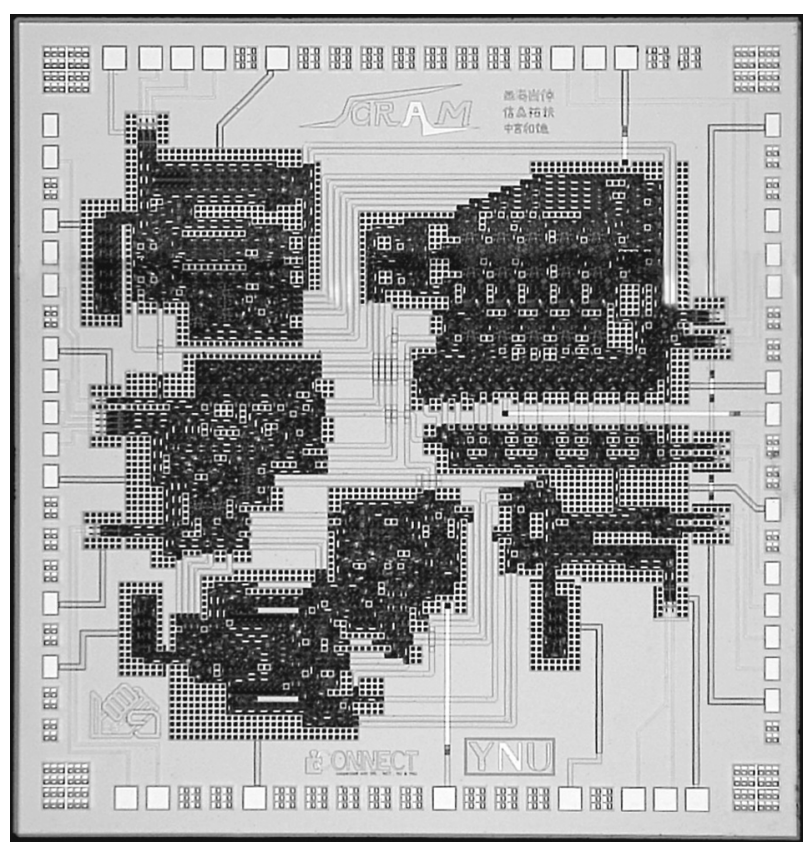

Fig. 5. Microphotograph of the SCRAM2. Die size is $5 \mathrm{~mm} \times 5 \mathrm{~mm}$.

1 to stage 2 is performed when HS1 detects both REQ1 and ACK2, which are completion signals from stage 1 and 2, respectively. In the same way, the transition from stage 2 to stage 3 occurs when REQ2 and ACK3 are detected by HS2. This handshaking enables a simple design for timing when implementing the pipelining.

The instruction set of SCRAMS is composed of eight instructions, which include data transfers (LD, ST), register operations (ADD, MOVE), conditional/unconditional branches (SKZ, JMP), a stop operation (HLT), and no-operation (NOP).

\section{IMPLEMENTATION AND PERFORMANCE EVALUATION}

We have designed and implemented the SCRAM2 using the CONNECT cell library [10] and the SRL $2.5 \mathrm{kA} / \mathrm{cm}^{2} \mathrm{Nb}$ standard process [11]. The target local clock frequency is $20 \mathrm{GHz}$. The system was first designed using the QDI model and the performance was then enhanced based on the SDI model. A micrograph of the SCRAM2 is shown in Fig. 5, where the number of Josephson junctions and the area of the circuit are 8197 and
TABLE I

COMPARISON OF MiCROPROCESSOR PERFORMANCE

\begin{tabular}{cccc}
\hline System & Cycle time [ ps ] & Performance [MIPS] & Over Head/Cycle time [\%] \\
\hline CORE1 $\alpha$ & 5000 & 200 & 16 \\
SCRAM2 QDI & 1918 & 521 & 8 \\
SCRAM2 SDI & 1733 & 577 & \\
\hline
\end{tabular}

TABLE II

DC Bias Margin For SEVERAl Circuit Components. ALU, Reg0, REg1, AND IR aRe MEasured at $20 \mathrm{GHz}$ By ON-CHIP High-SPEED TeSTS. PC IS MEASURED AT LOW SPEED

\begin{tabular}{clc}
\hline Component & \multicolumn{1}{c}{ Operation } & DC bias margin [\%] @20GHz \\
\hline ALU, Reg0, Reg1 & LD-MOVE-LD-ADD-ST & $+/-6.2$ \\
ALU, Reg0, Reg1 & LD-MOVE-LD-SKZ & $+/-21.7$ \\
IR & & $+/-13.6$ \\
PC & SKZ-JMP-JMP & $+/-11.2$ \\
\hline
\end{tabular}

$4.10 \times 3.74 \mathrm{~mm}^{2}$, respectively. The total bias current is $1.04 \mathrm{~A}$, which is fed through 17 bias current lines. The total power consumption is estimated to be $2.61 \mathrm{~mW}$.

We have evaluated the average performance of the SCRAM2 by logic simulation using a test program, which includes all instructions of the microprocessor. The estimated performance for different microprocessor designs is listed in Table I with the ratio of the handshaking overhead to cycle time. The performance of the SCRAM2, based on the SDI model, is approximately three times better than that of $\operatorname{CORE} 1 \alpha$, due to the introduction of asynchronous three-stage pipelining. Note that the SDI model has achieved a $10 \%$ performance improvement when compared with the QDI model, while the handshaking overhead is reduced by $50 \%$.

\section{Test Results}

Before investigating the functionality of the whole system we have individually tested the main circuit components of the SCRAM2. ALU with Reg0 and Reg1, IR and PC were implemented separately and tested by on-chip high-speed test systems [4]. Table II lists the DC bias margin of each circuit component at $20 \mathrm{GHz}$. Note that PC is measured only at low speed. Correct operations were confirmed for all components with sufficient DC bias margins.

The function of the whole system was then examined. Because the size of IM is four bytes, due to the restriction of the chip area, we have tested the operation of the system using four-instruction sequences as follows:

- JMP-NOP-NOP-HLT

- SKZ (Not Zero)-NOP-NOP-HLT

- SKZ (Zero)-NOP-NOP-HLT

- LD-ADD-ST-HLT

- LD-MOVE-ST-HLT,

These sequences check all instructions of the SCRAM2.

Fig. 6 shows the measurement results of the JMP-NOPNOP-HLT operation. The figure shows the output waveform from PC. It can be seen that the data in PC is incremented by 


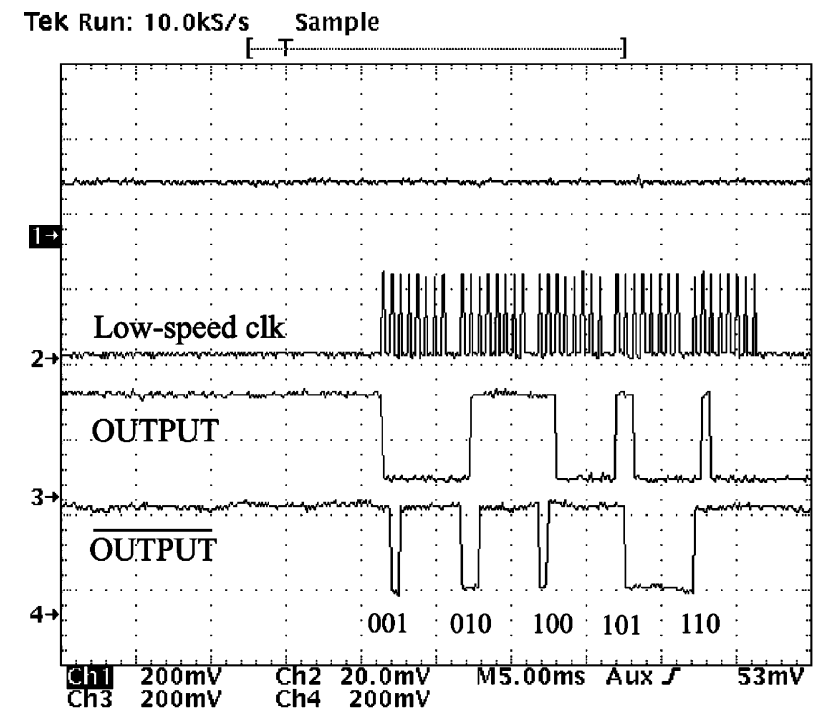

Fig. 6. Output waveforms from PC for a JMP-NOP-NOP-HLT operation. Note that a part of the handshaking circuit is disabled, and the pipeline stage is forwarded by an external low-speed system clock in this test for evaluation of the system states.

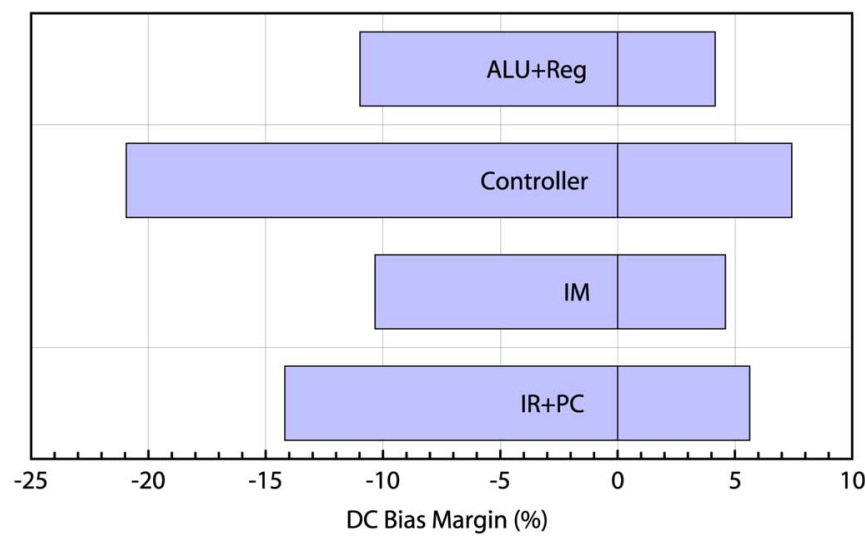

Fig. 7. DC bias margins of each circuit component in SKZ (Zero)-NOPNOP-HLT operation.

1 from 001 at every execution of an instruction, and jumps to 100 when JMP is executed. The correct operation of SKZ (Not Zero)-NOP-NOP-HLT and SKZ (Zero)- NOP-NOP-HLT were also confirmed. Fig. 7 shows the DC bias margins of each circuit component when the SKZ (Zero)-NOP-NOP-HLT operation is performed.

We have also measured operations, LD-ADD-ST-HLT and LD-MOVE-ST-HLT, but the correct operation was not observed, due to a malfunction of ALU. We suspect that the malfunction of ALU is caused by several reasons including flux trapping, defects in the circuits, and a large bias current that induces large magnetic fields.

\section{CONCLUSION}

We have designed a fully asynchronous 8-bit bit-serial RSFQ microprocessor, SCRAM2, based on asynchronous design approaches: data-driven self-timing, binary-decision-diagram logic and bit-serial handshaking. The performance of the microprocessor is enhanced based on the scalable-delay-insensitive model. The logic simulation indicated that the performance of the SCRAM2 is three times better than that of our previously developed synchronous RSFQ microprocessor CORE1 $\alpha$. The SCRAM2 was implemented using the CONNECT cell library and the SRL $2.5 \mathrm{kA} / \mathrm{cm}^{2} \mathrm{Nb}$ process. Correct operation of all circuit components was confirmed at $20 \mathrm{GHz}$, and the functionality of some instruction sequences were demonstrated in the whole system.

\section{REFERENCES}

[1] K. K. Likharev and V. K. Semenov, "RSFQ logic/family: A new Josephson-junction technology for sub-terahertz-clock-frequency digital systems," IEEE Trans. Appl. Supercond., vol. 1, pp. 3-28, Mar. 1991.

[2] H. Hayakawa, N. Yoshikawa, S. Yorozu, and A. Fujimaki, "Superconducting digital electronics," Proceedings of the IEEE., vol. 92, pp. 1549-1563, Oct. 2004.

[3] M. Tanaka, F. Matsuzaki, T. Kondo, N. Nakajima, Y. Yamanashi, A. Fujimaki, H. Hayakawa, N. Yoshikawa, H. Terai, and S. Yorozu, "A single-flux-quantum logic prototype microprocessor," in Technical Digest of IEEE International Solid-State Circuits Conference (ISSCC), San Francisco, USA, Feb. 2004, pp. 298-299.

[4] Z. J. Deng, N. Yoshikawa, S. R. Whiteley, and T. Van Duzer, "Datadriven self-timed RSFQ digital integrated circuit and system," IEEE Trans. Appl. Supercond., vol. 7, pp. 3634-3637, June 1997.

[5] N. Yoshikawa, H. Tago, and K. Yoneyama, "A new design approach for RSFQ logic circuits based on the binary decision diagram," IEEE Trans. Appl. Supercond., vol. 9, pp. 3161-3164, June 1999.

[6] M. Ito, K. Kawasaki, N. Yoshikawa, A. Fujimaki, H. Terai, and S. Yorozu, " $20 \mathrm{GHz}$ operation of bit-serial handshaking systems using asynchronous SFQ logic circuits," IEEE Trans. Appl. Supercond., vol. 15, pp. 255-258, June 2005.

[7] S. Hauck, "Asynchronous design methodologies: An overview," Proceedings of the IEEE, vol. 83, pp. 69-93, January 1995.

[8] A. Takamura, M. Kuwako, M. Imai, T. Fujii, M. Ozawa, I. Fukasaku, Y. Ueno, and T. Nanya, "TITAC-2: An asynchronous 32-bit microprocessor based on scalable-delay-insensitive model," in Proceedings of 1997 IEEE International Conference on Computer Design: VLSI in Computers and Processors, 1997. ICCD '97, Oct. 12-15, 1997, pp. 288-294.

[9] A. Fujimaki, Y. Takai, and N. Yoshikawa, "High-end server based on complexity-reduced architecture for superconductor technology," IEICE Trans. Electronics, vol. E85-C, no. 3, pp. 612-616, 2002.

[10] S. Yorozu, Y. Kameda, H. Terai, A. Fujimaki, T. Yamada, and S Tahara, "A single flux quantum standard logic cell library," Physica $C$ vol. 378-381, pt. 2, pp. 1471-1474, Oct. 2002.

[11] S. Nagasawa, Y. Hashimoto, H. Numata, and S. Tahara, "A 380 ps 9.5 $\mathrm{mW}$ Josephson 4-kbit RAM operated at a high bit yield," IEEE Trans. Appl. Supercond., vol. 5, pp. 2447-2452, June 1995. 